\title{
Comparação de Diferentes Modelos para Avaliação Genética de Características de Desempenho Pós-desmama em Suínos ${ }^{1}$
}

\author{
Fabiano Veraldo da Costa Pita², Lucia Galvão de Albuquerque ${ }^{3,4}$
}

\begin{abstract}
RESUMO - O objetivo deste trabalho foi avaliar diferentes modelos em que os efeitos aleatórios de leitegada e genético materno (correlacionado ou não com o efeito genético aditivo direto) foram adicionados em seqüência ao efeito aleatório genético aditivo direto. Foram utilizados registros de desempenho de 17.563 animais Landrace (LD) e 8679 Large White (LW), submetidos a teste de granja. A comparação dos modelos e a significância de cada efeito adicionado foi realizada por intermédio do teste da Razão de Verossimilhança. Os componentes de variância foram estimados pelo método da Máxima Verossimilhança Restrita. Para a raça LD o melhor modelo incluiu os efeitos genéticos direto e materno, além do efeito de leitegada. Para a raça LW o melhor modelo incluiu os efeitos genético direto e de leitegada. As estimativas de herdabilidade para ganho de peso médio diário, idade ao final do teste e número de dias de permanência no teste foram, respectivamente, $0,28,0,24$ e 0,24 para a raça $L D$, e $0,28,0,27$ e 0,23 para a raça $L W$. O efeito de leitegada foi entre 0,08 e 0,12 para ambas as raças. O efeito materno na raça $L D$ foi de magnitude muito pequena $(0,01$ a 0,03$)$. As correlações entre os efeitos genéticos direto e materno foram nulas ou negativas.
\end{abstract}

Palavras-chave: efeito de leitegada, efeito materno, ganho de peso médio diário, idade ao final do teste

\section{Comparison of Different Models for Genetic Evaluation of Post-weaning Performance Traits in Swine}

\begin{abstract}
The aim of this work was to evaluate different models in which the random effects of litter and maternal genetic (correlated or not with the direct additive genetic effect) were added in sequence to the direct additive genetic random effect. There were on farm records of 17,563 Landrace (LD) and 8679 Large White (LW) animal. The comparison of the models and significance levels of each effect added were determined by the Likelihood Ratio test. Variance components were estimated using the Restricted Maximum Likelihood method. For LD breed the best model included the direct and maternal genetic effects, besides the litter effect. For the LW breed the best model included the direct genetic and litter effects. Heritability estimates of average daily gain, off-test age, and number of days on test were, respectively, $0.28,0.24$, and 0.24 for LD breed, and $0.28,0.27$, and 0.23 for LW breed. The litter effect was between 0.08 and 0.12 for both breeds. The maternal effect in LD breed was of small magnitude ( 0.01 to 0.03$)$. Correlations between direct and maternal genetic effects were null or negative.
\end{abstract}

Key Words: average daily gain, litter effect, maternal effect, off-test age

\section{Introdução}

A estimação acurada do valor genético dos indivíduos submetidos a um programa de melhoramento genético depende, em grande parte, dos efeitos considerados no modelo estatístico utilizado para a avaliação dos animais.

No caso particular da avaliação genética de suínos, a covariância existente entre indivíduos de uma mesma família resulta, além dos genes em comum que estes indivíduos possuem, do ambiente comum que os animais de uma mesma leitegada compartilham durante o período pré-desmame. Este ambiente comum afeta o desempenho dos indivíduos em toda a sua vida e é determinado, principalmente, pela própria matriz, através dos seus cuidados com a cria, sua produção de leite etc.

AHLSCHWEDE e ROBISON (1971), ao realizarem um experimento envolvendo troca de leitões entre fêmeas, observaram que 14 a $19 \%$ da variância total para ganho de peso pós-desmame, peso aos 140 dias de idade e espessura de toucinho era devida à mãe biológica e 9 a $12 \%$ à fêmea que amamentou os leitões, evidenciando a importância da habilidade materna sobre características de desempenho. Essa habilidade materna em proporcionar à cria um am-

\footnotetext{
${ }^{1}$ Parte da dissertação de mestrado do primeiro autor (Curso de pós-graduação em Zootecnia, área de concentração em Genética e Melhoramento Animal, Faculdade de Ciências Agrárias e Veterinárias, UNESP/Campus de Jaboticabal), financiada pela FAPESP.

2 Zootecnista. Endereço atual: Universidade Federal de Viçosa, Curso de pós-graduação em Genética e Melhoramento. E.mail: fvcpita@bol.com.br

3 Professora assistente doutora. FCAV - UNESP/Jaboticabal. Depto. de Zootecnia, setor de Melhoramento Animal. E.mail: Igalb@fcav.unesp.br

4 Pesquisadora do CNPq.
} 
biente apropriado ao seu desenvolvimento é determinada por fatores ambientais e pelos genes que a mãe possui, os quais são transmitidos à sua progênie e se expressam apenas em seus descendentes fêmeas (efeito genético materno).

Na literatura é bastante comum a consideração do efeito de ambiente permanente e comum (efeito de leitegada) nos modelos para análise de características de desempenho em suínos, não ocorrendo o mesmo para o efeito genético materno. FERRAZ e JOHNSON (1993) utilizaram diferentes modelos, em relação aos efeitos aleatórios considerados, para a análise do ganho de peso médio diário (GMD) de suínos Landrace e Large White, não verificando, em ambas as raças, importância significativa do efeito genético materno para esta característica. Resultados semelhantes foram observados por CRUMP et al. (1997), ao avaliarem o GMD em suínos Landrace. Segundo estes últimos autores, a não significância deste efeito pode ter ocorrido devido à estrutura dos dados analisados. Por outro lado, BRYNER et al. (1992), ao avaliarem o GMD de cachaços Yorkshire, verificaram estimativas de herdabilidade e de efeito materno de, respectivamente, 0,24 e 0,23 , sendo a correlação genética entre estes efeitos de -0,23.

O objetivo do presente trabalho foi avaliar diferentes modelos, em relação aos efeitos aleatórios considerados, para a avaliação genética de características de desempenho em suínos Landrace e Large White.

\section{Material e Métodos}

\section{Dados}

Foram utilizadas informações de 17.563 animais Landrace e 8679 animais Large White, nascidos entre 1991 e 1998, e pertencentes ao núcleo de seleção de suínos da Granja Rezende S.A., que desenvolve atividades agropecuárias em Uberlândia (MG).

Os animais nasceram em instalações climatizadas e a desmama ocorreu com 14 dias de idade. Após a saída da creche (em torno de 64 dias de idade), os animais de cada sexo foram alojados, separadamente, em galpões de crescimento e terminação. Nestes galpões o teste de granja foi iniciado com a pesagem dos animais, após um período de adaptação de sete dias, e finalizado quando os animais atingiam 145 dias de idade. Tanto para as fêmeas quanto para os machos a ração foi servida à vontade em baias coletivas.

Os animais Landrace iniciaram e terminaram o teste compesos médios de, respectivamente, $25,16 \pm 4,64 \mathrm{~kg}$ e $90,70 \pm 10,49 \mathrm{~kg}$, enquanto que os animais Large White apresentaram $25,00 \pm 4,89 \mathrm{~kg}$ e $91,83 \pm 11,53 \mathrm{~kg}$, respectivamente. A estrutura dos dados analisados e de parentesco pode ser observada, respectivamente, nas Tabelas 1 e 2, para cada raça.

As características de desempenho analisadas foram: ganho de peso médio diário, do desmame ao abate, (GMD), idade para atingir um peso-padrão de $95 \mathrm{~kg}$ (IF95) e, número de dias de permanência no teste para atingir um peso-padrão de $95 \mathrm{~kg}$ (ND95). As médias e desvios-padrão para GMD foram $0,87 \pm 0,10$ e $0,91 \pm 0,12 \mathrm{~kg} / \mathrm{dia}$, para IF95 foram $151,05 \pm 13,43$ e $149,26 \pm 13,87$ dias de idade e para ND95, 82,07 $\pm 13,16$ e 78,49 $\pm 13,86$ dias, para os animais Landrace e Large White, respectivamente.

\section{Análise estatística}

As três características foram analisadas para cada raça separadamente, utilizando-se quatro modelos em que diferentes efeitos aleatórios foram adicionados em seqüência. Os modelos utilizados foram:

Modelo $1-\mathrm{y}=\mathrm{X} \beta+\mathrm{Z}_{1} \mathrm{~g}+\mathrm{e}$;

Modelo $2-\mathrm{y}=\mathrm{X} \beta+\mathrm{Z}_{1} \mathrm{~g}+\mathrm{Wc}+\mathrm{e}$;

Modelo $3-\mathrm{y}=\mathrm{X} \beta+\mathrm{Z}_{1} \mathrm{~g}+\mathrm{Z}_{2} \mathrm{~m}+\mathrm{Wc}+\mathrm{e}, \mathrm{com} \mathrm{r}_{\mathrm{gm}}=0$; Modelo $4-\mathrm{y}=\mathrm{X} \beta+\mathrm{Z}_{1} \mathrm{~g}+\mathrm{Z}_{2} \mathrm{~m}+\mathrm{Wc}+\mathrm{e}, \mathrm{com} \mathrm{r}_{\mathrm{gm}} \neq 0$. em que: $\mathbf{y}$, é o vetor das observações; $\boldsymbol{\beta}$, o vetor dos efeitos fixos; $\mathbf{g}$, o vetor dos efeitos genéticos aditivos diretos dos animais; $\mathbf{m}$, o vetor dos efeitos genéticos maternos dos animais; $\mathbf{c}$, o vetor dos efeitos permanentes e comuns a membros da mesma leitegada; $\mathbf{e}$, o vetor dos efeitos residuais; $\mathbf{r}_{\mathbf{g m}}$, a correlação gené-

Tabela 1 - Estrutura dos dados analisados para cada raça Table 1 - Analyzed data structure for each breed

\begin{tabular}{|c|c|c|c|c|}
\hline $\begin{array}{l}\text { Raça } \\
\text { Breed }\end{array}$ & $\begin{array}{c}\mathrm{N}^{\mathrm{o}} \text { de animais } \\
N \text {. of animals }\end{array}$ & $\begin{array}{l}\mathrm{N}^{\circ} \text { de animais na } \mathrm{A}^{1} \\
N \text {. of animals in the } A^{1}\end{array}$ & $\begin{array}{c}\mathrm{N}^{\mathrm{o}} \text { de } \mathrm{GC} \mathrm{C}^{2} \mathrm{~s}^{2} \\
N . \text { of } C G^{\prime} s^{2}\end{array}$ & $\begin{array}{c}\mathrm{N}^{\mathrm{o}} \text { de leitegadas } \\
\text { N. of litters }\end{array}$ \\
\hline Landrace & 17.563 & 17.905 & 305 & 2708 \\
\hline Large White & 8679 & 8894 & 213 & 1342 \\
\hline
\end{tabular}

${ }^{1}$ A - matriz de parentesco; ${ }^{2} \mathrm{GC}$ - grupo contemporâneo.

${ }^{1} A$ - relationship matrix; ${ }^{2} C G$ - contemporary group. 
Tabela 2 - Estrutura de parentesco para cada raça

Table 2 - Relationship structure for each breed

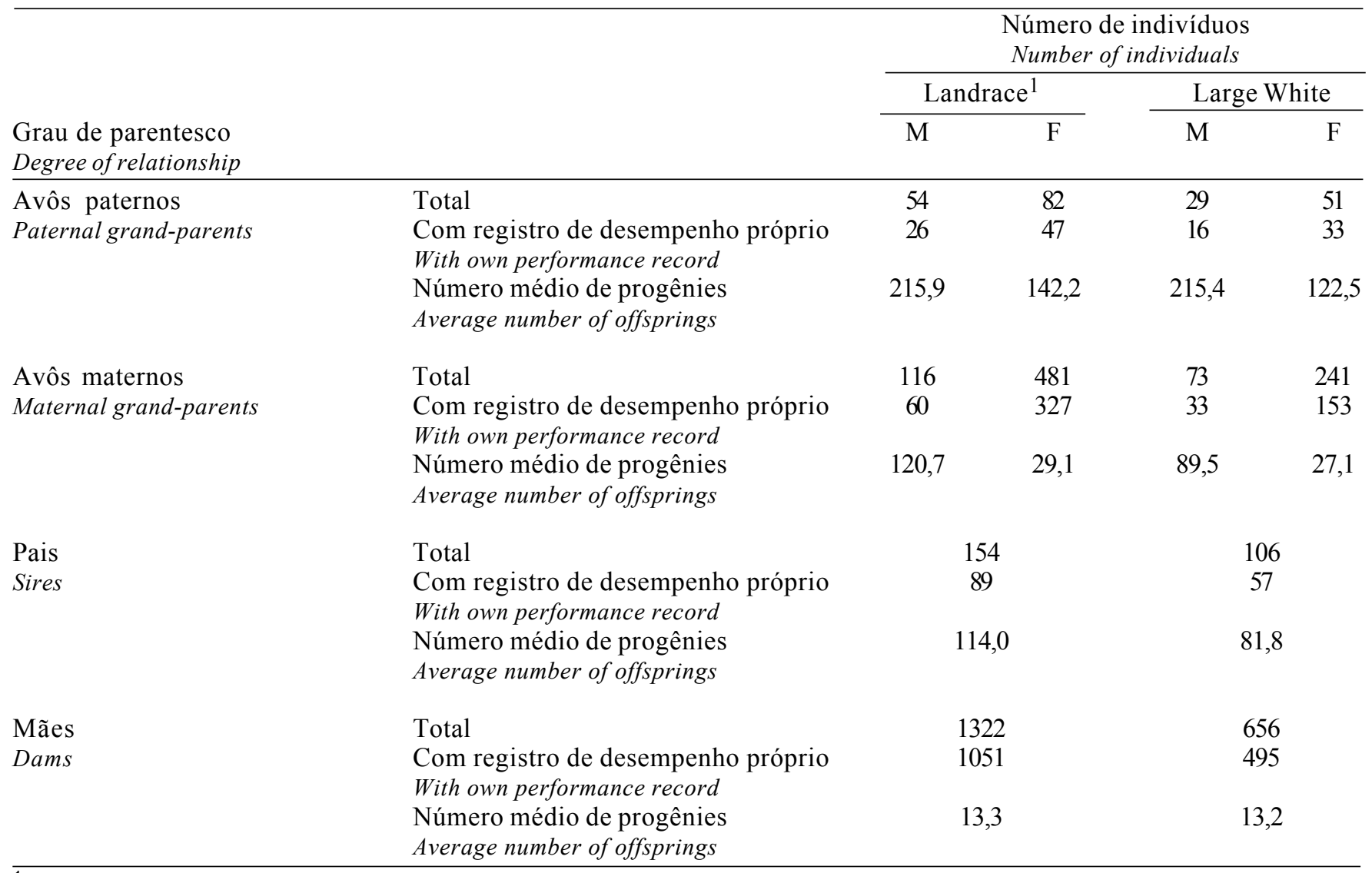

${ }^{1} \mathrm{M}$ - macho, $\mathrm{F}$ - fêmea ( $M$ - male, $F$ - female).

tica entre $\mathrm{g}$ e $\mathrm{m}$ e, $\mathrm{X}, \mathrm{Z}_{1}, \mathrm{Z}_{2}$ e $\mathrm{W}$ são matrizes de incidência que associam os efeitos apropriados a $\mathrm{y}$.

As pressuposições para os modelos foram:

$\mathrm{E}[\mathrm{y}]=\mathrm{Xb} ; \mathrm{E}[\mathrm{g}]=0 ; \mathrm{E}[\mathrm{m}]=0 ; \mathrm{E}[\mathrm{c}]=0 ; \mathrm{E}[\mathrm{e}]=0$; $\operatorname{Var}[\mathrm{g}]=A \sigma_{\mathrm{g}}^{2} ; \operatorname{Var}[\mathrm{m}]=A \sigma_{\mathrm{m}}^{2} ; \operatorname{Var}[\mathrm{c}]=\mathrm{I}_{\mathrm{NL}} \sigma^{2}{ }_{\mathrm{c}}$; $\operatorname{Var}[\mathrm{e}]=\mathrm{I}_{\mathrm{N}} \sigma_{\mathrm{e}}^{2} ; \operatorname{Cov}[\mathrm{g}, \mathrm{m}]=0$ (modelo 3$)$, $\operatorname{Cov}[\mathrm{g}, \mathrm{m}]=\mathrm{A} \sigma_{\mathrm{gm}}($ modelo 4$)$

em que: $\mathrm{NL}=$ número de leitegadas; $\sigma_{\mathrm{g}}{ }^{2}=$ variância genética aditiva direta; $\mathrm{N}=$ número de registros; $\sigma_{\mathrm{m}}{ }^{2}$ $=$ variância genética materna; $\mathrm{A}=$ numerador da matriz de parentesco; $\sigma_{\mathrm{c}}{ }^{2}=$ variância de ambiente permanente e comum (leitegada); I = matriz identidade; $\sigma_{\mathrm{e}}^{2}=$ variância residual; $\sigma_{\mathrm{gm}}=$ covariância genética aditiva direta e materna.

As covariâncias entre os efeitos genéticos e ambientais (permanentes e temporários) bem como entre os efeitos ambientais de diferentes animais foram assumidos como zero.

Os efeitos fixos incluídos nos modelos foram: a) grupo contemporâneo (GC), definido como animais de mesmo sexo, local, mês e ano de nascimento e grupo de teste; b) idade ao início do teste e peso ao início do teste, como covariáveis, para análise do GMD e ND95, respectivamente. A consideração destas características como covariáveis baseou-se em análises anteriores (PITA, 2000).

Para melhor estimação do efeito de GC e visando-se evitar o confundimento deste efeito com o de leitegada foram impostas, durante a edição dos dados, restrições quanto ao número mínimo de animais e de leitegadas por GC (mínimo de 7 animais e 4 leitegadas, respectivamente).

Os componentes de (co)variância foram estimados pelo método de Máxima Verossimilhança Restrita (REML) não derivativo, aplicado a modelos animais, utilizando-se o programa computacional MTDFREML (BOLDMAN et al., 1993), que, em vez de maximizar o logaritmo neperiano da função de verossimilhança (ln L), adota o procedimento de minimizar o $-2 \ln \mathrm{L}$. O ponto de mínimo do $-2 \ln \mathrm{L}$ foi 
avaliado usando-se o método Simplex, assumindo-se a convergência das iterações quando a variância dos valores de $-2 \ln \mathrm{L}$ foi menor que $10^{-9}$.

A comparação entre os modelos e a significância de cada efeito incluído no modelo foi verificada por intermédio do teste da Razão de Verossimilhança (HOGG e CRAIG, 1995), comparando-se a redução no $-2 \ln \mathrm{L}$, causada pela adição de um parâmetro ao modelo, a uma distribuição Qui-quadrado com um grau de liberdade.

\section{Resultados e Discussão}

$\mathrm{Na}$ Tabela 3 podem ser observadas, para ambas as raças, a redução na estimativa do $-2 \ln \mathrm{L}$ e a significância desta redução, causada pela utilização de diferentes modelos, para a análise de cada uma das características de desempenho.

Conforme pode ser observado na Tabela 3, em ambas as raças e para as três características analisadas houve redução significativa do $-2 \ln \mathrm{L}$ estimado quando o efeito aleatório de ambiente permanente e comum (c) foi incluído no modelo de análise (modelo 2), em relação ao modelo inicial (modelo 1) que considerava como aleatório apenas o efeito genético aditivo direto dos animais (g). A significância do efeito de leitegada indica que este efeito responde por uma porção importante da variação existente na população para as características de desempenho consideradas. Portanto, espera-se que sua inclusão no modelo de análise genética permita a predição mais acurada do valor genético dos indivíduos sob avaliação. FERRAZ e JOHNSON (1993) e CRUMP et al. (1997), também observaram significância do efeito de leitegada para o ganho de peso médio diário.

Quando o efeito aleatório genético materno $(\mathrm{m})$ foi adicionado aos efeitos g e c (modelo 3 ) e comparado ao modelo 2 , verificou-se redução significativa do $-2 \ln \mathrm{L}$ estimado para todas as características analisadas na raça Landrace. Já para a raça Large White, a inclusão de $m$ não diminuiu a função de máxima verossimilhança significativamente.

FERRAZ e JOHNSON (1993) e CRUMP et al. (1997), ao analisarem o ganho de peso médio diário, também não observaram significância do efeito genético materno para a raça Large White. De acordo com estes últimos autores, a não significância deste efeito pode estar relacionada com a estrutura dos dados analisados, já que o número de gerações contempladas pela estrutura de parentesco disponível era pequeno.

Neste estudo, a não significância do efeito genético materno observada na raça Large White pode estar relacionada com a quantidade de informação

Tabela 3 - Redução nas estimativas do -2 In L e sua significância causada pela utilização de diferentes modelos para a análise do ganho de peso médio diário (GMD), idade (IF95) e número de dias (ND95) para atingir $95 \mathrm{~kg}$ de peso vivo, para as raças Landrace e Large White

Table 3 - Reduction in estimates of -2 In $L$ and its significance, caused by utilization of different models for analyzing average daily gain (ADG), off-test age (A95), and number of days on test (ND95) to $95 \mathrm{~kg}$ of live weight, for Landrace and Large White breeds

\begin{tabular}{|c|c|c|c|c|}
\hline \multirow[b]{2}{*}{$\begin{array}{l}\text { Raça } \\
\text { Breed }\end{array}$} & \multirow[b]{2}{*}{$\begin{array}{c}\text { Característica } \\
\text { Trait } \\
\end{array}$} & \multicolumn{3}{|c|}{$\begin{array}{l}\text { Redução do }-2 \ln \mathrm{L}^{1} \text { estimado } \\
\text { Reduction in the }-2 \ln L^{1} \text { estimated }\end{array}$} \\
\hline & & $\begin{array}{l}\text { Modelo1-Modelo2 } \\
\text { Model1-Model2 }\end{array}$ & $\begin{array}{l}\text { Modelo2-Modelo3 } \\
\text { Model2-Model3 }\end{array}$ & $\begin{array}{l}\text { Modelo3-Modelo4 } \\
\text { Model3-Model4 }\end{array}$ \\
\hline \multirow[t]{3}{*}{ Landrace } & $\begin{array}{l}\text { GMD } \\
A D G\end{array}$ & $284,55 * *$ & $11,88 * *$ & $1,87 \mathrm{NS}$ \\
\hline & $\begin{array}{l}\text { IF95 } \\
A 95\end{array}$ & $304,16 * *$ & $30,79 * *$ & $0,33 \mathrm{NS}$ \\
\hline & $\begin{array}{l}\text { ND95 } \\
\text { ND95 }\end{array}$ & $300,44 * *$ & $3,992 *$ & $6,70 * *$ \\
\hline \multirow[t]{3}{*}{ Large White } & $\begin{array}{l}\text { GMD } \\
A D G\end{array}$ & $172,43 * *$ & $1,91 \mathrm{NS}$ & $2,95 \mathrm{NS}$ \\
\hline & $\begin{array}{l}\text { IF95 } \\
A 95\end{array}$ & $217,99 * *$ & $3,29 \mathrm{NS}$ & $0,00 \mathrm{NS}$ \\
\hline & $\begin{array}{l}\text { ND95 } \\
\text { ND95 }\end{array}$ & $160,65 * *$ & $1,13 \mathrm{NS}$ & $0,00 \mathrm{NS}$ \\
\hline
\end{tabular}

${ }^{* *} P<0,01,{ }^{*} P<0,05, N S$ - não significativo.

$1 \mathrm{~L}=$ função de verossimilhança.

${ }^{* *} P<0.01,{ }^{*} P<0.05, N S-$ not significative.

$1 \mathrm{~L}=$ likelihood function.

Rev. bras. zootec., 30(6):1720-1727, 2001 
disponível. Isso porque os genes responsáveis por este efeito são expressos apenas nos indivíduos fêmeas e, portanto, a determinação e avaliação de sua importância talvez dependa da existência, no conjunto de dados, de um considerável número de parentes fêmeas com progênie avaliada.

A Tabela 2 mostra que a proporção de avôs maternos e de mães com registro de desempenho próprios é semelhante para ambas as raças (aproximadamente 59 e $65 \%$ para avôs e 75 e $79 \%$ para mães, respectivamente para as raças Large White e Landrace). Mas, em termos absolutos, a quantidade de informação existente para a raça Landrace proveniente destes indivíduos é duas vezes maior que na raça Large White. Ainda, a raça Landrace possui mais machos que têm filhas com progênies avaliadas (avôs maternos) e estes avôs, por sua vez, têm um maior número médio de progênies que os avôs maternos na raça Large White.

Quando os efeitos $\mathrm{g}$ e $\mathrm{m}$ foram considerados correlacionados (modelo 4) houve redução significativa do - 2 ln L estimado, em relação ao modelo 3, apenas para a característica ND95, quando analisada na raça Landrace. Este resultado pode ser devido à estrutura dos dados, como será discutido mais adiante.

Nas Tabelas 4 e 5 podem ser observadas, respectivamente para as raças Landrace e Large White, as estimativas de parâmetros genéticos e fenotípicos obtidas utilizando-se os quatro diferentes modelos de análise propostos.

Para a raça Landrace verificou-se que, à exceção de quando o modelo de análise foi o modelo 1 , as estimativas de herdabilidade $\left(\mathrm{h}^{2}\right)$, de efeito materno $\left(\mathrm{m}^{2}\right)$ e de efeito de leitegada $\left(\mathrm{c}^{2}\right)$ foram bastante próximas para as três características analisadas (entre 0,24 e 0,32 para $^{2}$, entre 0,01 e 0,03 para $\mathrm{m}^{2}$ e, entre 0,08 e 0,10 para $\mathrm{c}^{2}$ ). Quando o modelo 1 foi utilizado as estimativas de $\mathrm{h}^{2}$ para as três características foram maiores que 0,40 . As correlações genéticas entre os efeitos genéticos aditivo direto e materno $\left(\mathrm{r}_{\mathrm{gm}}\right)$ foram negativas para as três características, embora tenham diferido em magnitude $(-0,26,-0,10$ e -0,52, respectivamente para GMD, IF95 e ND95).

As estimativas de herdabilidade para GMD na raça Landrace obtidas com o modelo 3 foram similares às observadas por KAPLON et al. (1991), CARDELLINO e SIEWERDT (1992), ROSO (1997a) e HERMESCH et al. (1998) e inferiores à verificadas por LO et al. (1992) e SILVA et al. (1992). A magnitude das estimativas de $\mathrm{m}^{2}$ foram semelhantes às relatadas por FERRAZ e JOHNSON (1993), CRUMP et al. (1997) e PANETO (1998), mas inferiores às observadas por BRYNER et al. (1992). As estimativas de $\mathrm{c}^{2}$ obtidas concordaram com as observadas por FERRAZ e JOHNSON (1993) e CRUMP et al. (1997), sendo bem inferiores às relatadas por HOFER et al. (1992).

Tabela 4 - Estimativas de parâmetros genéticos e fenotípicos para as características ganho de peso médio diário (GMD), idade (IF95) e número de dias (ND95) para atingir $95 \mathrm{~kg}$ de peso vivo, analisadas na raça Landrace e utilizando-se os quatro modelos propostos

Table 4 - Estimates of genetic and phenotypic parameters for average daily gain (ADG), off-test age (A95), and number of days on test (ND95) to $95 \mathrm{~kg}$ of live weight, for the Landrace breed using the four models proposed

\begin{tabular}{|c|c|c|c|c|c|c|}
\hline \multirow[b]{2}{*}{$\begin{array}{l}\text { Característica } \\
\text { Trait }\end{array}$} & \multirow[b]{2}{*}{$\begin{array}{c}\text { Modelo } \\
\text { Model }\end{array}$} & \multicolumn{5}{|c|}{$\begin{array}{l}\text { Parâmetros genéticos e fenotípicos }{ }^{1} \\
\text { Genetic and phenotypic parameters }{ }^{1}\end{array}$} \\
\hline & & $h^{2}$ & $\mathrm{~m}^{2}$ & $\mathrm{r}_{\mathrm{gm}}$ & $c^{2}$ & $\mathrm{e}^{2}$ \\
\hline GMD & 1 & 0,44 & - & - & - & 0,56 \\
\hline \multirow[t]{3}{*}{$A D G$} & 2 & 0,32 & - & - & 0,09 & 0,59 \\
\hline & 3 & 0,28 & 0,02 & - & 0,09 & 0,61 \\
\hline & 4 & 0,32 & 0,03 & $-0,26$ & 0,08 & 0,59 \\
\hline IF95 & 1 & 0,44 & - & - & - & 0,56 \\
\hline \multirow[t]{3}{*}{ A95 } & 2 & 0,30 & - & - & 0,10 & 0,60 \\
\hline & 3 & 0,24 & 0,03 & - & 0,08 & 0,65 \\
\hline & 4 & 0,25 & 0,03 & $-0,10$ & 0,08 & 0,64 \\
\hline ND95 & 1 & 0,40 & - & - & - & 0,60 \\
\hline \multirow[t]{3}{*}{ ND95 } & 2 & 0,26 & - & - & 0,09 & 0,65 \\
\hline & 3 & 0,24 & 0,01 & - & 0,09 & 0,66 \\
\hline & 4 & 0,32 & 0,03 & $-0,52$ & 0,09 & 0,62 \\
\hline
\end{tabular}

${ }_{1} \mathrm{~h}^{2}=$ herdabilidade; $\mathrm{m}^{2}=$ efeito materno; $\mathrm{r}_{\mathrm{gm}}=$ correlação genética entre efeito genético aditivo direto e efeito genético materno; $\mathrm{c}^{2}=$ efeito de leitegada; $\mathrm{e}^{2}=$ resíduo.

${ }^{1} h^{2}=$ heritability; $m^{2}=$ maternal effect; $r_{g m}=$ genetic correlation between direct and maternal genetic effect; $c^{2}=$ litter effect; $e^{2}=$ error. 
As estimativas obtidas para IF95 utilizando-se o modelo 3 foram bastante inferiores àquelas verificadas por KENNEDY et al. (1985), LO et al. (1992), ROSO et al. (1995), e ROSO (1997b), mas maiores que as relatadas por BRAZÃO (1997). Estimativas semelhantes foram observadas por BIDANEL e DUCOS (1996) e TORRES JR. et al. (1998). Para ND95, ROSO (1997b) descreveu estimativas de herdabilidade similares às observadas neste estudo.

Para a raça Large White verificaram-se estimativas mais baixas de $\mathrm{h}^{2}$ para a característica ND95 (entre 0,21 e 0,23 ) em relação às observadas para GMD e IF95 (entre 0,24 e 0,33 ), quando estas foram analisadas através de modelos que incluíam o efeito de leitegada (modelos 2 a 4). Quando o modelo 1 foi utilizado, estimativas de $\mathrm{h}^{2}$ próximas e superiores (entre 0,38 e 0,44 ) foram observadas para as três características. Independentemente do modelo, as estimativas de $\mathrm{m}^{2}$ e $\mathrm{c}^{2}$ foram bastante semelhantes para as três características. As estimativas de $\mathrm{r}_{\mathrm{gm}}$ para IF95 e ND95 foram zero, enquanto que para GMD esta foi maior em magnitude $(-0,45)$ que a observada para a raça Landrace, embora a consideração desta covariância no modelo de análise não contribuiu para a redução significativa do $-2 \ln \mathrm{L}$.

MERKS (1988), HOFER et al. (1992) e BRAZÃO (1997) relataram estimativas de $h^{2}$ para GMD inferiores às observadas neste trabalhando utilizando-se omodelo 2. Já estimativas similares foram verificadas por KERR e
CAMERON (1996) e BRANDT e TÄUBERT (1998). A estimativa de $\mathrm{c}^{2}$ para esta característica foi semelhante à relatada por DUCOS et al. (1993) e inferior à observada por HOFER et al. (1992). A estimativa de herdabilidade para IF95 utilizando-se o modelo 2 foi similar às observadas por VAN DIEPEN e KENNEDY (1989), BIDANEL e DUCOS (1996) e TORRES JR. et al. (1998), mas inferior às observadas por SILVA et al. (1992) e LI e KENNEDY (1994) e superior à estimada por BRAZÃO (1997). Os resultados obtidos para $\mathrm{c}^{2}$ foram semelhantes aos observados por BIDANEL et al. (1994), KERR e CAMERON (1996) e TORRES JR. et al. (1998). Para ND95, as estimativas de herdabilidade e efeito de leitegada utilizando-se o modelo 2 foram similares às verificadas por KERR e CAMERON (1996).

As estimativas nulas ou negativa observadas para $\mathrm{r}_{\mathrm{gm}}$ foram semelhantes às obtidas por BRYNER et al. (1992), FERRAZ e JOHNSON (1993) e CRUMP et al. (1997). Quando estas estimativas não foram nulas, as estimativas de $\mathrm{h}^{2} \mathrm{e}^{2}$ foram diferentes das obtidas com o modelo de análise 3. Estes resultados concordaram com os obtidos por MEYER (1997) para gado de corte, que observou que estimativas de alta magnitude e negativas para a covariância entre os efeitos genéticos direto e materno estavam associadas com superestimação dos efeitos genéticos aditivo direto e materno.

Poucos trabalhos discutem as estimativas de $r_{\text {gm }}$ para

Tabela 5 - Estimativas de parâmetros genéticos e fenotípicos para as características ganho de peso médio diário (GMD), idade (IF95) e número de dias (ND95) para atingir $95 \mathrm{~kg}$ de peso vivo, analisadas na raça Large White e utilizando-se os quatro modelos propostos

Table 5 - Estimates of genetic and phenotypic parameters for average daily gain (ADG), off-test age (A95), and number of days on test (ND95) to $95 \mathrm{~kg}$ of live weight, for the Large White breed using the four models proposed

\begin{tabular}{lcccccc}
\hline & & \multicolumn{5}{c}{$\begin{array}{c}\text { Parâmetros genéticos e fenotípicos } \\
\text { Genetic and phenotypic parameters }\end{array}$} \\
\cline { 2 - 6 } $\begin{array}{l}\text { Característica } \\
\text { Trait }\end{array}$ & $\begin{array}{c}\text { Modelo } \\
\text { Model }\end{array}$ & $\mathrm{h}^{2}$ & $\mathrm{~m}^{2}$ & $\mathrm{r}_{\mathrm{gm}}$ & $\mathrm{c}^{2}$ & $\mathrm{e}^{2}$ \\
\hline GMD & 1 & 0,43 & - & - & - & 0,57 \\
ADG & 2 & 0,28 & - & - & 0,11 & 0,61 \\
& 3 & 0,26 & 0,01 & - & 0,10 & 0,63 \\
& 4 & 0,33 & 0,04 & $-0,45$ & 0,10 & 0,58 \\
IF95 & 1 & 0,44 & - & - & - & 0,56 \\
A95 & 2 & 0,27 & - & - & 0,12 & 0,61 \\
& 3 & 0,24 & 0,02 & -11 & 0,63 \\
ND95 & 4 & 0,24 & 0,02 & 0,00 & 0,11 & 0,63 \\
ND95 & 1 & 0,38 & - & - & - & 0,62 \\
& 2 & 0,23 & - & - & 0,10 & 0,67 \\
& 3 & 0,21 & 0,01 & 0,10 & 0,68 \\
& 4 & 0,21 & 0,01 & 0,00 & 0,10 & 0,68 \\
\hline
\end{tabular}

${ }^{1} \mathrm{~h}^{2}=$ herdabilidade; $\mathrm{m}^{2}=$ efeito materno; $\mathrm{r}_{\mathrm{gm}}=$ correlação genética entre efeito genético aditivo direto e efeito genético materno; $\mathrm{c}^{2}=$ efeito de leitegada; $\mathrm{e}^{2}=$ resíduo

${ }^{1} h^{2}=$ heritability; $m^{2}=$ maternal effect; $r_{g m}=$ genetic correlation between direct and maternal genetic effect; $c^{2}=$ litter effect; $e^{2}=$ error.

Rev. bras. zootec., 30(6):1720-1727, 2001 
características de desempenho em suínos. AHLSCHWEDE e ROBISON (1971), estudando a influência do efeito materno sobre características de crescimento e espessura de toucinho em suínos verificaram covariâncias positivas entre os efeitos genéticos aditivo direto e materno para peso e ganho de peso, medidos semanalmente, durante o primeiro mês de vida dos leitões. Após a quarta semana, estes efeitos se tornaram correlacionados negativamente. De acordo com os autores, estes resultados podem ser explicados pelo fato de leitões amamentados por fêmeas com pouca produção de leite procurarem suplementação mais precocemente que aqueles cujas mães possuem uma grande produção de leite, sendo que esta suplementação mais que compensa a amamentação adicional. Isto poderia explicar a estimativa de correlação negativa e de alta magnitude observada neste trabalho para a raça Landrace.

Alguns autores têm sugerido que estas estimativas negativas e de média a alta magnitude para $r_{g m}$ podem estar relacionadas com o tamanho do conjunto de dados, com a sua estrutura e, a magnitude dos efeitos aditivos direto e materno. Segundo GERSTMAYR (1992), os componentes mais importantes da estrutura dos dados para a estimação do efeito genético materno são o número de progênies por fêmea e o número de fêmeas com registro de desempenho próprio no conjunto de dados. Em situações onde nenhuma das fêmeas têm registro de desempenho próprio, a $r_{g m}$ é estimada utilizando-se apenas o parentesco entre os indivíduos avaliados. GERSTMAYR (1992) também estudou a influência da estrutura dos dados e da magnitude dos efeitos genéticos direto e materno sobre a confiabilidade das estimativas de $r_{\mathrm{gm}}$. Estes aspectos foram investigados através de simulação de diferentes conjuntos com diferentes estruturas, em relação ao número médio de progênies por fêmeas e proporção de fêmeas sem registro de desempenho próprio, e diferentes parâmetros genéticos verdadeiros. $\mathrm{O}$ autor verificou que a confiabilidade das estimativas de $r_{g m}$ foi fortemente prejudicada quando a população simulada possuía fêmeas sem registro de desempenho próprio ou com apenas uma progênie por fêmea. Entretanto, a maior influência sobre a confiabilidade das estimativas foi devida à proporção entre os componentes de variância genético aditivo direto e materno nas populações simuladas. A confiabilidade das estimativas de $r_{g m}$ foi menor nas populações onde o efeito genético materno era quatro vezes menor que o efeito genético aditivo direto. $\mathrm{O}$ autor concluiu que conjuntos de dados maiores e melhor estruturados são necessários para se obter estimativas de $r_{\text {gm }}$ mais confiáveis quando a variância genética materna é menor ou de similar magnitude que a variância genética aditiva direta. Neste trabalho, os efeitos genéticos maternos foram dez vezes menores que os efeitos genéticos aditivos diretos e, portanto, as estimativas de $\mathrm{r}_{\mathrm{gm}}$ aqui obtidas podem não ser confiáveis.

O efeito sobre a predição dos valores genéticos aditivo direto e materno dos indivíduos devido à inclusão nos modelos de análise da $r_{g m}$ quando esta não pode ser bem estimada foi estudado por BENYSHEK et al. (1988), que utilizaram-se de três conjuntos de dados de bovinos de corte sob seleção simulados com valores verdadeiros de $r_{\text {gm }}$ negativo, nulo ou positivo. Ao analisarem cada conjunto simulado considerando-se uma $r_{\text {gm }}$ negativa, nula ou positiva os autores verificaram que a acurácia de predição do valor genético aditivo direto não foi afetada pela $\mathrm{r}_{\mathrm{gm}}$ utilizada para análise, não ocorrendo o mesmo para a acurácia de predição do valor genético materno quando $r_{g m}$ negativa foi utilizada para analisar o conjunto simulado com $r_{\text {gm }}$ positiva e vice-versa. Quando a $r_{\text {gm }}$ utilizada para análise era nula a acurácia destas estimativas de valor genético era bastante boa, levando os autores a concluírem que a utilização de $\mathrm{r}_{\mathrm{gm}}$ nula quando estimativas acuradas de seu valor verdadeiro não estão disponíveis permite a obtenção acurada de valores genéticos aditivo direto e materno.

\section{Conclusões}

O efeito de leitegada é uma importante fonte de variação para as características de desempenho em suínos no pós-desmama, devendo ser considerada nos modelos de avaliação genética a fim de obter-se predições acuradas dos valores genéticos dos indivíduos. Já a importância do efeito materno parece ser mais dependente da estrutura do conjunto de dados sob avaliação. Ainda, neste trabalho a correlação entre os efeitos genéticos direto e materno foi importante apenas para o ND95 na raça Landrace. Entretanto, considerando-se as dificuldades para obtenção de estimativas confiáveis para $r_{g m}$, assumir esta como nula talvez seja o melhor procedimento para a avaliação genética de características em que o efeito genético materno é importante, mas de pequena magnitude.

Mais estudos são necessários para identificar as causas de correlações negativas entre os componentes genéticos de características de desempenho em suínos, avaliadas após a desmama. 


\section{Agradecimento}

À FAPESP (Fundação de Amparo à Pesquisa do Estado de São Paulo), pelo financiamento desta pesquisa e, à Granja Rezende S.A., pelo fornecimento dos dados utilizados neste estudo.

\section{Referências Bibliográficas}

AHLSCHWEDE, W.T., ROBISON, O.W. 1971. Prenatal and postnatal influences on growth and backfat in swine. J. Anim. Sci., 32:10-16.

BENYSHEK, L.L., JOHNSON, M.H, LITTLE, D.E. et al. 1988. Applications of an animal model in the United States beef cattle industry. J. Dairy Sci., 71(Suppl.2):35-53.

BIDANEL, J.P., DUCOS, A., GUÉBLEZ, R. et al. 1994. Genetic parameters of backfat thickness, age at $100 \mathrm{~kg}$ and ultimate $\mathrm{pH}$ in on-farm tested French Landrace and Large White pigs. Livest. Prod. Sci., 40:291-301.

BIDANEL, J.P., DUCOS, A. 1996. Genetic correlations between test station and on-farm performance traits in Large White and French Landrace pig breeds. Livest. Prod. Sci., 45:55-62.

BOLDMAN, K.G., KRIESE, L.A., VAN VLECK, L.D. et al. 1993. A manual for use of MTDFREML. USDA-ARS, Lincoln, USA.

BRANDT, H., TÄUBERT, H. 1998. Parameter estimates for purebred and crossbred performances in pigs. J. Anim. Breed. Genet., 115:97-104.

BRAZÃO, C.S. Estimativas de parâmetros genéticos e ambientais para algumas características de desempenho em suínos da raça Large White. Jaboticabal, SP: FCAV/UNESP, 1997. 90p. Dissertação (Mestrado em Zootecnia) - Faculdade de Ciências Agrárias e Veterinárias/Universidade Estadual Paulista, 1997.

BRYNER, S.M., MABRY, J.W., BERTRAND, J.K. et al. 1992. Estimation of direct and maternal heritability and genetic correlation for backfat and growth rate in swine using data from centrally tested Yorkshire boars. J. Anim. Sci., 70:1755-1759.

CARDELLINO, R.A., SIEWERDT, F. 1992. Genetic parameters of production traits in Landrace and Large White pigs in southern Brazil. Rev. Bras. Genet., 15:575-583.

CRUMP, R.E., HALEY, C.S., THOMPSON, R. et al. 1997. Individual animal model estimates of genetic parameters for performance test traits of male and female Landrace pigs tested in a commercial nucleus herd. Anim. Sci., 65:275-283.

DUCOS, A., BIDANEL, J.P., DUCROCQ, V. et al. 1993. Multivariate restricted maximum likelihood estimation of genetic parameters for growth, carcass and meat quality traits in French Large White and French Landrace pigs. Genet. Sel. Evol. 25:475-493.

FERRAZ, J.B.S., JOHNSON, R.K. 1993. Animal model estimation of genetic parameters and response to selection for litter size and weight, growth, and backfat in closed seedstock populations of Large White and Landrace Swine. J. Anim. Sci., 71:850-858.

GERSTMAYR, S. 1992. Impact of the data structure on the reliability of the estimated genetic parameters in an animal model with maternal effects. J. Anim. Breed. Genet., 109:321-336.

HERMESCH, S., LUXFORD, B.G., GRASER, H.U. Genetic relationships of growth and lean meat with meat quality and reproduction traits in Australian pigs. In: WORLD CONGRESS ON GENETICS APPLIED TO LIVESTOCK PRODUCTION, 6, 1998, Armidale. Proceedings... Armidale, 1998. p.511-514.

HOFER, A., HAGGER, C., KÜNZI, N. 1992. Genetic evaluation of on-farm tested pigs using an animal model. I. Estimation of variance components with restricted maximum likelihood. Livest. Prod. Sci., 30:69-82.

HOGG, R.V., CRAIG, A.T. 1995. Introduction to mathematical statistics. Prentice Hall, New Jersey, USA. 527p.

KAPLON, M.J., ROTHSCHILD, M.F., BERGER, P.J. et al. 1991. Population parameter estimates for performance and reproductive traits in Polish Large White nucleus herds. J. Anim. Sci., 69:91-98.

KENNEDY, B.W., JOHANSSON, K., HUDSON, G.F.S. 1985. Heritabilities and genetic correlations for backfat and age at $90 \mathrm{~kg}$ in performance tested pigs. J. Anim. Sci., 61:78-82.

KERR, J.C., CAMERON, N.D. 1996. Genetic and phenotypic relationships between performance test and reproduction traits in Large White pigs. Anim. Sci., 62:531-540.

LI, X.W., KENNEDY, B.W. 1994. Genetic parameters for growth rate and backfat in Canadian Yorkshire, Landrace, Duroc and Hampshire pigs. J. Anim. Sci., 72:1450-1454.

LO, L.L., MCLAREN, D.G., MCKEITH, F.K. et al. 1992. Genetic analyses of growth, real-time ultrasound, carcass, and pork quality traits in Duroc and Landrace pigs: IIHeritabilities and correlations. J. Anim. Sci., 70:2387-2396.

MERKS, J.W.M. 1988. Genotype X environment interactions in pig breeding programmes.III. Environmental effects and genetic parameters in on-farm test. Livest. Prod. Sci., 18:129-140.

MEYER, K. 1997. Estimates of genetic parameters for weaning weight of beef cattle accounting for direct-maternal environmental covariances. Livest. Prod. Sci., 52:187-197.

PANETO, J.C.C. Comparações entre as tendências genéticas e econômicas de um rebanho elite de suínos. Jaboticabal, SP: FCAV/UNESP, 1998. 90p. Dissertação (Mestrado em Zootecnia) - Faculdade de Ciências Agrárias e Veterinárias / Universidade Estadual Paulista, 1998.

PITA, F.V.C. Modelos para avaliação genética e comparação de características de desempenho para a seleção de suínos. Jaboticabal, SP: FCAV, 2000. 157p. Dissertação (Mestrado em Zootecnia) - Faculdade de Ciências Agrárias e Veterinárias / Universidade Estadual Paulista, 2000.

ROSO, V.M. Parâmetros genéticos para ganho de peso médio diário e espessura de toucinho em suínos de diferentes grupos raciais. In: REUNIÃO ANUAL DA SOCIEDADE BRASILEIRA DE ZOOTECNIA, 34, 1997, Juiz de Fora. Anais... Juiz de Fora: SBZ, 1997a. p.332-334.

ROSO, V.M. Alternativas para avaliar taxa de crescimento e conseqüências na seleção de suínos. In: REUNIÃO ANUAL DA SOCIEDADE BRASILEIRA DE ZOOTECNIA, 34, 1997, Juiz de Fora. Anais..., Juiz de Fora: SBZ, 1997b. p.308-310.

ROSO, V.M., FRIES, L.A., MARTINS, E.S. 1995. Parâmetros genéticos em características de desempenho e qualidade de carcaça em suínos da raça Duroc. R. Soc. Bras. Zootec., 24:310-316.

SILVA, M.A., CATALAN, G., TORRES, R.A. et al. 1992. Estimativas de componentes genéticos de características de importância econômica, em três diferentes raças de suínos. R. Soc. Bras. Zootec., 21:923-932.

TORRES JR., R.A.A., SILVA, M.A., LOPES, P.S. et al. 1998. Estimativas de componentes de (co)variância para características produtivas de suínos Landrace e Large White pelo método da máxima verossimilhança restrita. Rev. bras. zootec., 27:283-291.

VAN DIEPEN, T.A., KENNEDY, B.W. 1989. Genetic correlations between test station and on-farm performance for growth rate and backfat in pigs. J. Anim. Sci., 67:1425-1431.

Recebido em: 07/02/01 Aceito em: 08/05/01 